\title{
FRAMES AND OPERATORS IN HILBERT $C^{*}$-MODULES
}

\author{
Abbas Najati, M. Mohammadi Saem and P. GĂvruţa
}

Abstract. In this paper we introduce the concepts of atomic systems for operators and $K$-frames in Hilbert $C^{*}$-modules and we establish some results.

Mathematics subject classification (2010): 42C15, 46L05, 46H25.

Keywords and phrases: Atomic system, $K$-frame, $C^{*}$-algebra, Hilbert $C^{*}$-module, Bessel sequence.

\section{REFERENCES}

[1] Lu. ARAmbašIĆ, On frames for countably generated Hilbert $C^{*}$-modules, Proc. Amer. Math. Soc. 2 (135) (2007), 469-478.

[2] D. BAKIĆ, B. GulJaš, Hilbert $C^{*}$-modules over $C^{*}$-algebras of compact operators, Acta Sci. Math(Szeged), 1-2 (68) (2002), 249-269.

[3] O. Christensen, An introduction to frames and Riesz bases, Birkhäuser, Boston-Basel-Berlin, 2002.

[4] R. J. Duffin And A. C. Schaeffer, A class of nonharmonic Fourier series, Trans. Amer. Math. Soc. (72) (1952), 341-366.

[5] X. FANG, J. YU, H. YAO, Solutions to operator equations on Hilbert $C^{*}$-modules, Linear Algebra. Appl, 11 (431) (2009) 2142-2153.

[6] M. Frank, D. R. LARSOn, A module frame concept for Hilbert $C^{*}$-modules, The functional and harmonic analysis of wavelets and frames (San Antonio, TX, 1999), Contemp. Math., (247) (1999), 207-233.

[7] M. Frank, D. R. Larson, Frames in Hilbert $C^{*}$-modules and $C^{*}$-algebras, J. Operator Theory, 2 (48) (2002), 273-314.

[8] L. GĂ VRuţA, Frames for operators, App. Comput. Harmon. Anal. 1 (32) (2012), 139-144.

[9] L. GĂVRUŢA, Atomic decomposition for operators in reproducing kernel Hilbert spaces, Mathematical Reports, accepted.

[10] G. G. Kasparov, Hilbert $C^{*}$-modules: theorems of Stinespring and Voiculescu, J. Operator Theory 4 (1) (1980), 133-150.

[11] H. LI, A Hilbert $C^{*}$-module admitting no frames, Bull. London Math. Soc. 42 (3) (2010), 388-394.

[12] W. Jing, Frames in Hilbert $C^{*}$-modules, Ph.D. Thesis, University of Central Frorida. 2006.

[13] E. C. LANCE, Hilbert $C^{*}$-modules: A toolkit for operator algebraists, London Mathematical Society Lecture Note Series 210, Cambridge University Press, Cambridge, 1995.

[14] V. M. Manuilov, E. V. Troitsky, Hilbert $C^{*}$-Modules, Translations of Mathematical Monographs, Vol. 226, AMS, Providence, Rhode Island, 2005.

[15] W. PASCHKE, Inner product modules over $B^{*}$-algebras, Trans. Amer. Math. Soc., (182) (1973), $443-$ 468.

[16] X. Xiao, Y. Zhu, L. Găvruţa, Some properties of $K$-frames in Hilbert spaces, Results Math. 3-4 (63) (2013) 1243-1255. 